\title{
Universal nonexponential relaxation: Complex dynamics in simple liquids
}

\author{
David A. Turton ${ }^{\text {a) }}$ and Klaas Wynne \\ Department of Physics, SUPA, University of Strathclyde, Glasgow G4 ONG, United Kingdom
}

(Received 29 September 2009; accepted 29 October 2009; published online 30 November 2009)

\begin{abstract}
The dynamics of the noble-gas liquids underlies that of all liquids making them an important prototypical model system. Using optical Kerr-effect spectroscopy we show that for argon, krypton, and xenon, both the librational and diffusional contributions to the spectrum are surprisingly complex. The diffusional relaxation appears as a stretched-exponential, such as widely found in studies of structured (e.g., glass-forming) liquids and as predicted by mode-coupling theory. We show that this behavior is remarkably similar to that measured in water and suggest that it is a fundamental or universal property. (C) 2009 American Institute of Physics. [doi:10.1063/1.3265862]
\end{abstract}

Argon has the simplest interatomic potential of any liquid allowing the most accurate simulations to be made and consequently there have been extensive neutron and $\mathrm{x}$-ray scattering studies. ${ }^{1-4}$ Such techniques are directly sensitive to atomic positions and fluctuations in structure, and are therefore particularly appropriate for the study of the phononlike modes in the terahertz region but are less informative of the lower frequency diffusive dynamics. Ultrafast optical Kerreffect (OKE) spectroscopy probes many-body interactions and is established as a powerful probe of structural relaxation in both complex and simple liquids. ${ }^{5-12}$ However, no detailed OKE study of the noble-gas liquids exists. OKE spectroscopy (the time-domain variant of Raman spectroscopy) measures the time-derivative of the two-point time-correlation of the anisotropic part of the many-body polarizability tensor. Consequently, the subterahertz spectra of molecular liquids are typically dominated by rotational modes. The translational modes, which are crucial to the transport properties that define chemical and biological reaction dynamics, generally make a weak contribution through the collisioninduced spectrum. Noble-gas liquids are therefore of particular interest as they are free of the rotational and intramolecular vibrations of molecular liquids and their spectra consist entirely of this, less well understood, collisioninduced, or interaction-induced contribution.

Our basic OKE setup has been described previously. ${ }^{12}$ The OKE response of these liquids is relatively weak, so sample purity is critical and as it was found that atmospheric gases, including nitrogen, are readily dissolved by the samples, especially argon, the apparatus was made gas-tight. A gradient-seal quartz cuvette (Starna Optiglass) was extended by fusing an additional $30 \mathrm{~cm}$ glass tube to the opening. The assembly was placed in a cryostat (Oxford Instruments, Optistat DN) with the tube extended through the top entry port to a gas line. The line was evacuated, and the sample gases (Sigma-Aldrich, $\geq 99.995 \%$ purity) then condensed directly into the cuvette.

The OKE data in the time domain for argon, krypton, and xenon are shown in Fig. 1. On the liquid-gas coexistence

${ }^{a)}$ Electronic mail: david.turton@phys.strath.ac.uk. curve the liquids are stable over only a few degrees of temperature and in this range no change of the OKE signal was detected. In each case then, the data were measured within one degree of the triple point. The model of behavior expected for a simple unstructured liquid might be a simple cage-rattling mode that evolves into a diffusive, probably exponential, decay. The measured signal is however far more complex. Following the instantaneous (hyperpolarizability) peak, the nuclear response initially rises and is followed by an oscillatory region (marked in Fig. 1 by a pair of inflexion points) that suggests some high-frequency complexity. The signal subsequently decays smoothly but is nonexponential.

The data for xenon are shown in detail in Fig. 2 and in the frequency domain in Fig. 3. Detailed analysis is not straightforward and has not been made previously. ${ }^{10,13}$ The Bucaro-Litovitz function was derived as a model of the Raman spectrum for these liquids ${ }^{14}$ but is based on (head-on) collisions in the gas phase for a Lennard-Jones fluid and is a poor model of this response failing, particularly, to reproduce

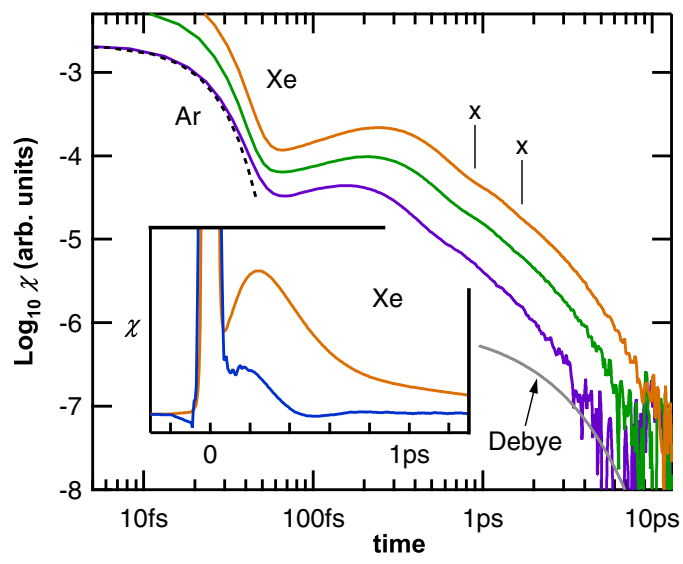

FIG. 1. Time-domain OKE data for argon (purple), krypton (green), and xenon (orange) displayed on logarithmic axes. Although the intensity units are arbitrary, the relative intensities are as measured and reflect the relative magnitude of the atomic polarizabilities. The data were measured, in each case, within $1 \mathrm{~K}$ of the triple point (see Table I). The instantaneous response is shown (dashed) and the two inflexions, for xenon, are marked $\times$. The nonexponential relaxation is apparent when compared with the exponential (Debye) function. Inset: detail on linear axes of the OKE data for crystalline xenon at $158 \mathrm{~K}$ (blue), compared with the liquid phase (orange). 


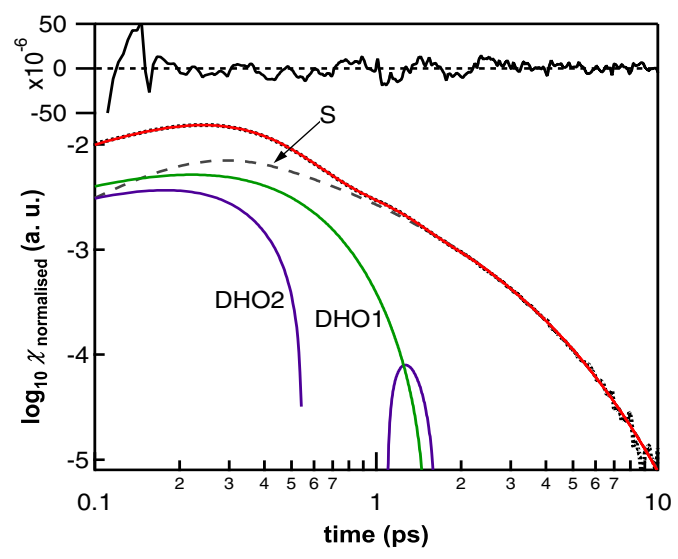

FIG. 2. Detail of the OKE data and fit for xenon. Up to $\sim 5 \mathrm{ps}$, the data (dots) are indistinguishable from the fit (red). Also shown are the stretchedexponential function S [Eq. (1)) (dashed)] and the pair of DHOs. Above are the fit residuals, which show that the fit is generally within the noise of the data. Very similar fits were obtained for argon and krypton, and the fit parameters for each liquid are shown in Table I.

the broad nature of the spectrum. The most complete model of relaxation is provided by mode-coupling theory (MCT) developed, and now widely applied, in studies of glass forming liquids. ${ }^{6,15-18} \mathrm{MCT}$ predicts a critical (singular) temperature $T_{C}$ typically $15 \%-20 \%$ above the glass-transition temperature. Above $T_{C}$, a low frequency $\alpha$ relaxation is observed that is diffusive and therefore temperature dependent. This is accompanied by temperature-independent, i.e., nondiffusive or oscillatory, fast dynamics. The lineshape of the $\alpha$ relaxation is typically stretched (with respect to a Debye function) but the lineshape and amplitude are temperature-independent above $T_{C}$. Stretching arises naturally in MCT from a feedback mechanism in which momentum is coupled into the cage structure thereby generating a distribution of relaxation timescales in which complete decay of the density fluctuations is prevented. The stretching has been associated widely with the presence of mesoscopic heterogeneity as expected for some glass forming liquids, however, MCT has also been

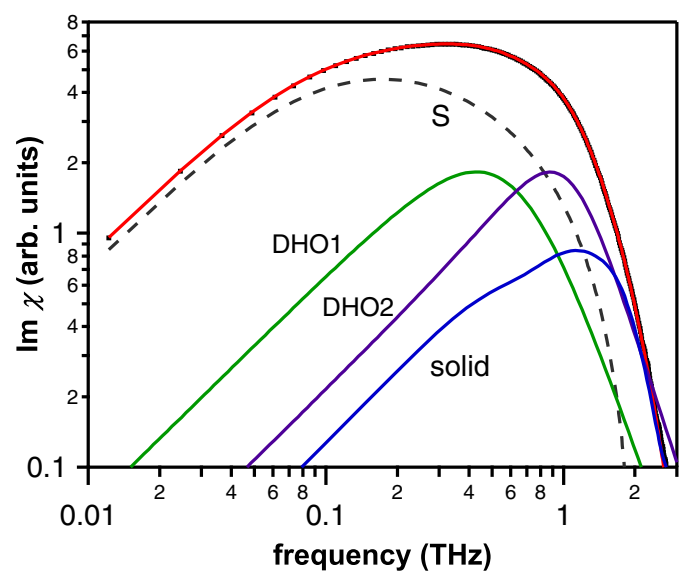

FIG. 3. The imaginary part of the OKE spectrum on logarithmic axes for xenon. The data (dots) are indistinguishable from the fit (red). The components of the fit are the Fourier transform of the stretched-exponential function S [Eq. (1)], (dashed), and the DHOs. Also shown is the spectrum of the solid xenon at $158 \mathrm{~K}$ (blue). applied to relatively simple liquids such as benzene ${ }^{19}$ and water. ${ }^{11,18}$

Although there has been some progress in extending MCT to include the librational modes of relatively simple liquids, ${ }^{19,20}$ there is not yet a practical model that can be used without a priori knowledge of the behavior and that can be interpreted in terms of the physical properties of the liquid. Consequently, practical applications have employed empirical models, such as the Cole-Davidson function or the derivative of the stretched exponential function $d / d t \exp [$ $\left.-(t / \tau)^{\beta}\right]$, which arises naturally from the MCT model, to describe the stretched $\alpha$ relaxation. ${ }^{11}$ In the absence of a temperature dependence we resort to a simple model to give some insight into the physical behavior. In Fig. 2 it can be seen that at times greater than $\sim 2 \mathrm{ps}$, the decay measured in xenon is consistent with the derivative of the stretchedexponential function with $\beta=0.66$. The fits to argon and krypton are very similar (Table I).

It is well known that Debye-like functions, particularly stretched functions, are unphysical at high frequency and, in scattering studies, should be modified by an inertial rise term. For this, the empirical function $1-\exp \left(-k_{\mathrm{RT}} t\right)$ has been used. ${ }^{5,12}$ However, in analyzing these data, we find that a steeper rise is necessary to be consistent with the oscillatory behavior at high frequency through which the anisotropy arises and here a Gaussian rise function is employed. As is seen in Fig. 2, the inertial rise function influences only the very short time behavior and allows the rise of the diffusional mode to correctly follow the librational dynamics. Although it is unsatisfactory to treat the relaxational modes as independent from the librations, this process has only a weak influence on the nature of the dynamics of interest here. The full expression for the diffusive part of the OKE decay is then

$$
\begin{aligned}
\chi_{S}(t)= & A_{S}\left(1-\exp \left[-k_{\mathrm{RT}}^{2} t^{2}\right]\right) \\
& \times \beta \tau^{-1} \exp \left[-(t / \tau)^{\beta}\right](t / \tau)^{\beta-1} .
\end{aligned}
$$

In the frequency domain, above $1 \mathrm{THz}$, a sharp cutoff is seen, which is much steeper than the falloff of a Debye function and marks the oscillatory behavior visible in the time domain. To fit the data accurately, a pair of oscillatory modes is necessary and the optimum fit was given by a pair of damped harmonic oscillators (DHOs) in addition to the diffusive mode. In the DHO model, ${ }^{21}$ coupling of the particle to the solvent fluctuations introduces an exponential damping factor. In the frequency domain, the function can be written as

$$
\chi_{B}(\omega)=A_{j} \omega_{j}^{2} /\left(\omega_{j}^{2}+i \gamma_{j} \omega-\omega^{2}\right),
$$

where oscillator $j$ with amplitude $A_{j}$, $\omega_{j}$ is the undamped frequency and $\gamma_{j}$ is the damping rate for which critical damping is achieved for $\gamma_{j}=2 \omega_{j}$.

Figure 2 shows detail of the fit for the xenon data; the fits for argon and krypton are very similar. The fit functions were generated in the frequency domain, but as no frequency-domain form of the stretched-exponential function exists, a numerical Fourier transform of Eq. (1) was summed with the oscillator functions, Eq. (2). The sum was then con- 
TABLE I. Fit parameters for argon, krypton, and xenon.

\begin{tabular}{lccccccccccc}
\hline \hline & $T$ & $A_{S}$ & $\beta$ & $\begin{array}{c}\tau \\
(\mathrm{ps})\end{array}$ & $\begin{array}{c}k_{\mathrm{RT}} \\
(\mathrm{THz})\end{array}$ & $\begin{array}{c}A_{1} \\
(\text { a.u. })\end{array}$ & $\begin{array}{c}\gamma_{1} / 2 \pi \\
(\mathrm{THz})\end{array}$ & $\begin{array}{c}\omega_{1} / 2 \pi \\
(\mathrm{THz})\end{array}$ & $\begin{array}{c}A_{2} \\
(\text { a.u. })\end{array}$ & $\begin{array}{c}\gamma_{2} / 2 \pi \\
(\mathrm{THz})\end{array}$ & $\begin{array}{c}\omega_{2} / 2 \pi \\
(\mathrm{THz})\end{array}$ \\
\hline $\mathrm{Xe}$ & 161 & 0.011 & 0.67 & 0.64 & 4.6 & 0.56 & 1.3 & 0.76 & 0.18 & 1.1 \\
$\mathrm{Kr}$ & 116 & 0.011 & 0.66 & 0.49 & 4.9 & 0.52 & 1.9 & 1.0 & 0.11 & 1.2 \\
$\mathrm{Ar}$ & 84 & 0.011 & 0.66 & 0.35 & 5.9 & 0.37 & 1.9 & 1.1 & 0.26 & 2.1 \\
\hline \hline
\end{tabular}

voluted with the autocorrelation function. For the experimental data, a 25 fs (full width at half maximum) Gaussian autocorrelation gave the closest fit. The resulting complex function was fitted to the raw data in the time domain by a standard Levenberg-Marquardt least-squares minimization through a numerical Fourier transform.

The fitted parameters for each sample are shown in Table I. The lower frequency oscillator is approximately critically damped while the higher is underdamped. The two oscillators are not resolved but without the second, narrower mode, the pair of inflexion points cannot be reproduced. There is a great deal of overlap between the three functions, but even with ten free parameters, there is good consistency between the three fits, particularly in the lineshapes. The (normalized) amplitudes and broadening parameters $\beta$ of the stretched-exponential mode are virtually identical. There is inevitable interaction between the parameters of the higher frequency modes, but their lineshapes are very similar. The underdamped oscillator DHO2 is closely defined by the high-frequency edge of the spectrum. The lower frequency oscillator DHO1 is necessarily more arbitrary. Notwithstanding this uncertainty in the oscillators, the frequencies and damping factors form a homologous series.

To understand this complexity it is useful to compare the model to the results of neutron and x-ray Brillouin scattering studies. ${ }^{3}$ Brillouin scattering experiments can measure the dispersion curves of the longitudinal acoustic (LA) and transverse acoustic (TA) phonon modes up to the Brillouin zone boundary in crystalline samples. In crystalline argon, the LA and TA phonon frequencies at the zone boundary are 1.6 and $2.1 \mathrm{THz} .^{22}$ In disordered materials such as liquids, spatial damping localizes the "phonons" near the pseudoBrillouin zone (PBZ) where the phonon wavelength becomes equal to the interatomic spacing. As a result, Brillouin scattering cannot resolve phonons near the PBZ.

The OKE signal in atomic liquids is wholly collision induced. The first-order collision-induced contribution $\overleftrightarrow{\pi}$ on atom $j$ due to a second atom $k$ can be expressed as

$$
\overleftrightarrow{\pi}_{j}=\overleftrightarrow{\alpha}_{j} \cdot \sum_{\substack{k=1 \\ k \neq j}}^{N} \stackrel{\leftrightarrow}{\mathrm{T}}_{j k} \cdot \overleftrightarrow{\alpha}_{k}
$$

where $\alpha$ is the isolated-atom polarizability and $\stackrel{\mathrm{T}}{j k}_{j}$ is the dipole-dipole interaction tensor. ${ }^{23}$ In atomic liquids, the dipole-dipole tensor is simply $\stackrel{\leftrightarrow}{\mathrm{T}}_{j k}=\overleftrightarrow{1} / r_{j k}^{3}$, where $r$ is the interatomic separation. Thus, the collision-induced spectrum depends on interatomic spacing as $r^{-6}$ to $r^{-8}$ and is therefore due to nearest neighbor contributions only. As a result, the
OKE spectrum represents the dynamics of (localized) phonons at the PBZ.

On freezing the noble-gas liquids, it is possible to form a metastable mixed phase of cubic and hexagonal close-packing $^{24}$ and on crystallization, one of our xenon samples remained sufficiently transparent for OKE measurements for several minutes before transforming into the microcrystalline state. In the solid phase, the activated (diffusive) modes freeze out and the remaining mode appears close to an underdamped Gaussian function (Fig. 3). Scattering artifacts lower the quality of the data preventing accurate fitting, but compared with $\mathrm{DHO} 2$ in the fit to liquid xenon the mode has a similar area, is also underdamped (Fig. 1), but moves to slightly higher frequency (peaking at $1.1 \mathrm{THz}$ from $0.88 \mathrm{THz}$ at $161 \mathrm{~K}$ ). This suggests that $\mathrm{DHO} 2$ is the residual of the localized LA phonon in the liquid state.

In Fig. 4, the signals for krypton and xenon have been rescaled to show that-despite the different properties of atomic mass, size, and polarizability - the decay function for the three atomic liquids is identical within the signal-to-noise ratio except for a pair of amplitude and frequency scaling parameters. Comparison of the amplitude parameter with a first order approximation of the relative amplitude $N \alpha^{2}$, where $N$ is the liquid number density at the triple point, ${ }^{25}$ and $\alpha$ is the static atomic polarizability, ${ }^{26}$ gives reasonable agreement suggesting that the amplitude scaling is trivial. The origin of the frequency scaling parameter is less simple in-

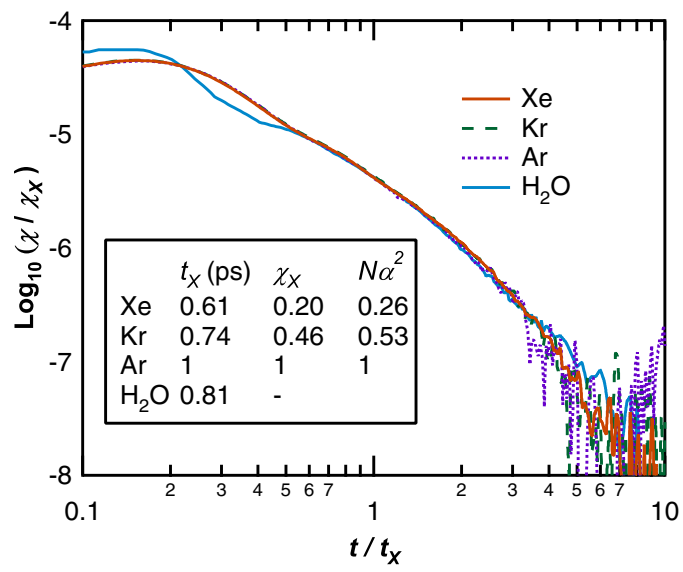

FIG. 4. Detail of the data for argon, krypton, and xenon with the amplitude and time axes for krypton and xenon rescaled to show the universal scaling. The inset table gives the scaling factors relative to argon. $N \alpha^{2}$ is, for comparison, an approximate amplitude factor based on the atomic polarizability (see text for details). Also shown are our OKE data for water for which the time-amplitude scaling applies only to the final diffusive decay due to the very different librational behavior of the strongly interacting liquid. These data were measured at an earlier date so the amplitude scaling cannot be compared. 
volving the mass, polarizability, and interatomic potential (which also determine the triple point). It is well known that the law of corresponding states ${ }^{27}$ applies to thermodynamic properties of simple liquids and it was long since demonstrated that the thermodynamic variables and phonon frequencies of noble gas solids are homologous and are related to each other through a scaling factor. ${ }^{28}$ Neutron inelastic scattering studies have also shown that the phonon dispersion curves for solid argon, krypton, and xenon ${ }^{29}$ and liquid neon and $\operatorname{argon}^{30}$ are scalable. Of all substances, argon, krypton, and xenon best satisfy the criteria for application of the law; here we see that it also applies to the dynamics in the liquid state.

The OKE response of water has only a weak rotational contribution so is also, predominantly, collision-induced. In Fig. 4, our OKE data for neat water is also shown. Before ca. $500 \mathrm{fs}$ the librational behavior is very different due to the familiar hydrogen-bonding modes, but it can be seen that the relaxational decay, over approximately two orders of magnitude in amplitude, exhibits the same stretched behavior as the atomic liquids.

Despite the apparent simplicity of the noble-gas liquids, the decay evolves from a pair of oscillatory modes into a stretched exponential relaxation. The OKE third-order response function cannot directly distinguish homogeneous from inhomogeneous line broadening mechanisms, however, its strength is that the high quality of the data allows detailed fitting and therefore interpretation through complex lineshapes. Here the two-DHO model gives a very precise fit to the data. Stretched $\alpha$ relaxation has generally been associated with heterogeneity, i.e., structure in liquids because of the ubiquity in supercooled and glass-forming liquids. In OKE studies of molecular liquids, the spectra are generally dominated by rotational modes and the translational diffusional modes are obscured. Here it is now clear that nonexponential "stretched" dynamics are present in the relaxations of simple liquids and appear therefore to be universal.

In a recent study of liquid and weakly supercooled water, ${ }^{11}$ the OKE relaxation was analyzed in the MCT framework and was also found to conform to the derivative of a stretched-exponential function with a very similar stretching parameter of $\beta \approx 0.6$ over the measured temperature range. This similarity suggests that the stretched exponential decay has a fundamental origin and whether it arises from structural inhomogeneity or is an intrinsic property of the trans- lational dynamics it supports the view that MCT is applicable to the entire liquid state rather than particularly to supercooled liquids.

We thank Peter Vöhringer for valuable discussion and acknowledge funding for this project from the Engineering and Physical Sciences Research Council (EPSRC).

${ }^{1}$ K. Sköld, J. M. Rowe, P. D. Randolph, and G. Ostrowsk, Phys. Rev. A 6, 1107 (1972).

${ }^{2}$ A. A. van Well, P. Verkerk, L. A. de Graaf, J. B. Suck, and J. R. D. Copley, Phys. Rev. A 31, 3391 (1985).

${ }^{3}$ J. B. Suck, Condens. Matter Phys. 11, 7 (2008)

${ }^{4}$ U. Bafile, F. Barocchi, and E. Guarini, Condens. Matter Phys. 11, 107 (2008)

${ }^{5}$ C. Kalpouzos, D. McMorrow, W. T. Lotshaw, and G. A. Kenneywallace, Chem. Phys. Lett. 150, 138 (1988).

${ }^{6}$ R. Torre, P. Bartolini, M. Ricci, and R. M. Pick, Europhys. Lett. 52, 324 (2000).

${ }^{7}$ K. Winkler, J. Lindner, and P. Vöhringer, Phys. Chem. Chem. Phys 4, 2144 (2002)

${ }^{8}$ H. Cang, V. N. Novikov, and M. D. Fayer, Phys. Rev. Lett. 90, 197401 (2003).

${ }^{9}$ H. Shirota, A. M. Funston, J. F. Wishart, and E. W. Castner, Jr., J. Chem. Phys. 122, 184512 (2005).

${ }^{10}$ N. H. Boeijenga, A. Pugzlys, T. L. C. Jansen, J. G. Snijders, and K. Duppen, J. Chem. Phys. 117, 1181 (2002).

${ }^{11}$ R. Torre, P. Bartolini, and R. Righini, Nature (London) 428, 296 (2004).

${ }^{12}$ D. A. Turton and K. Wynne, J. Chem. Phys. 128, 154516 (2008).

${ }^{13}$ B. I. Greene, P. A. Fleury, H. L. Carter, and R. C. Farrow, Phys. Rev. A 29, 271 (1984).

${ }^{14}$ J. A. Bucaro and T. A. Litovitz, J. Chem. Phys. 54, 3846 (1971).

${ }^{15}$ M. H. Ernst and J. R. Dorfman, J. Stat. Phys. 12, 311 (1975)

${ }^{16}$ W. Götze and L. Sjögren, Rep. Prog. Phys. 55, 241 (1992).

${ }^{17}$ E. Rössler, A. P. Sokolov, A. Kisliuk, and D. Quitmann, Phys. Rev. B 49, 14967 (1994)

${ }^{18}$ A. P. Sokolov, J. Hurst, and D. Quitmann, Phys. Rev. B 51, 12865 (1995).

${ }^{19}$ S. Wiebel and J. Wuttke, New J. Phys. 4, 56 (2002).

${ }^{20}$ V. Krakoviack and C. Alba-Simionesco, J. Chem. Phys. 117, 2161 (2002).

${ }^{21}$ Y. J. Yan and S. Mukamel, Phys. Rev. A 41, 6485 (1990).

${ }^{22}$ G. E. Moyano, P. Schwerdtfeger, and K. Rosciszewski, Phys. Rev. B 75, 024101 (2007).

${ }^{23}$ S. Ryu and R. M. Stratt, J. Phys. Chem. B 108, 6782 (2004).

${ }^{24}$ L. Meyer, C. S. Barrett, and P. Haasen, J. Chem. Phys. 40, 2744 (1964).

${ }^{25}$ Thermophysical Properties of Fluid Systems, edited by P. J. Linstrom and W. G. Mallard (National Institute of Standards and Technology, Gaithersburg, 2005).

${ }^{26}$ CRC Handbook of Chemistry and Physics, 87th ed., edited by D. R. Lide (Taylor \& Francis, London, 2007).

${ }^{27}$ E. A. Guggenheim, J. Chem. Phys. 13, 253 (1945).

${ }^{28}$ J. De Boer, Physica (Amsterdam) 14, 139 (1948).

${ }^{29}$ V. Ramamurthy and S. B. Rajendraprasad, J. Chem. Phys. 83, 3590 (1985).

${ }^{30}$ A. A. van Well and L. A. de Graaf, Phys. Rev. A 32, 2396 (1985). 